\title{
El doctor Pedro Morán, la Escuela Médica de 1833 y la batalla de Rancagua
}

\author{
Enrique Laval $R$.
}

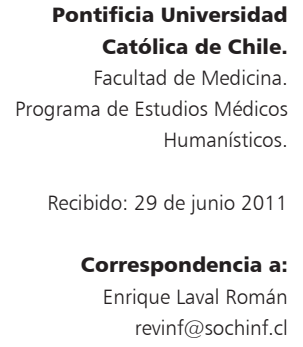

\author{
Dr. Pedro Morán, the Medical School of 1833, \\ and the Battle of Rancagua
}

With the foundation of the Medical School of 1833, the figure of Pedro Moran, the first professor of anatomy, physiology and hygiene, stands out. Diverse comments of his acts are recounted, some praising him and others revealing his concealment during the heat of the battle. His humble origins, competence and perseverance in the fulfilment of the teaching duties are extolled.

Key words: School of Medicine of 1833, Pedro Moran, Professor, military surgeon, Battle of Rancagua.

Palabras clave: Escuela Médica de 1833, Pedro Morán, profesor, cirujano militar, batalla de Rancagua.
Fundación de la escuela médica en 1833

4 n los primeros años de la década de 1830, ya estaban funcionando bastante bien los cursos de Leyes y Matemáticas en el Instituto Nacional. En cambio, el “curso médico” tardaría algo más en arraigarse, ya que hubo graves dificultades para su progreso, por parte de los alumnos y de los profesores.

Los jóvenes continuaban mirando a la medicina con desprecio y como una profesión inferior. Los docentes, que eran muy contados, pedían sueldos más altos o se negaban a dar el número de lecciones exigidas por los reglamentos.

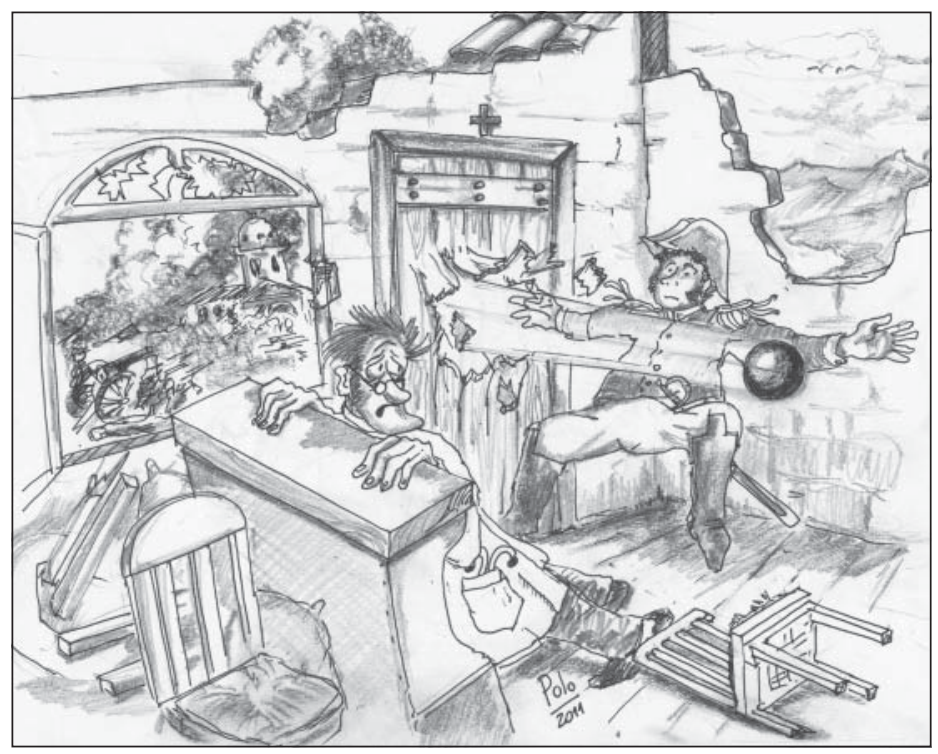

El 19 de marzo de 1833 el Gobierno de Joaquín Prieto, con la rúbrica de su Ministro Joaquín Tocornal, resolvió la creación de un "curso de estudios médicos en seis años": "deseando el Gobierno promover el estudio de las ciencias médicas que, aunque reconocidas en todas las naciones del mundo como de primera necesidad para la conservación de la vida, ha sido descuidado en Chile a influjo de una preocupación vulgar; y anhelando igualmente abrir una nueva y brillante carrera a la juventud estudiosa, ha venido en decretar y decreta:

Se abrirá en el Instituto Nacional un curso de ciencias médicas, que durará seis años, distribuidos en la forma siguiente:

Clase primera. Año primero. Anatomía especulativa y práctica. Año segundo. Continuación de la anatomía práctica, fisiología e higiene.

Clase segunda. Año primero. Principios y práctica de la medicina. Año segundo. Materia médica y medicina clínica en los hospitales. Año tercero. Los principios o práctica de cirugía y cirugía clínica. Año cuarto. Obstetricia y enfermedades incidentes a los niños.

\section{Comuníquese a quien corresponda.} Prieto. Tocornal”.

Este “curso de estudios médicos” sería dirigido por el doctor Guillermo Cunningham Blest, acompañado del doctor Pedro Morán, como profesor de anatomía, fisiología e higiene, de José Vicente Bustillos, que desde febrero de aquel año enseñaba farmacia y del doctor Lorenzo Sazie.

Comenzaron entonces los “balbuceos” de la Escuela Médica de 1833 en Chile ${ }^{1,2}$.

La medicina chilena durante los siglos anteriores, no conoció otra figura que la de fray Pedro Manuel Chaparro, fraile juandediano y médico, quien había sido el primero en variolizar en Chile e incluso en América (1765), realizando la primera vacunación anti-variólica en Santiago en 1805; poseedor de un sentido clínico admirable y dotado excepcionalmente para la docencia, a pesar de lo cual la Universidad de San Felipe lo había pospuesto para impartirla oficialmente ${ }^{3}$. 


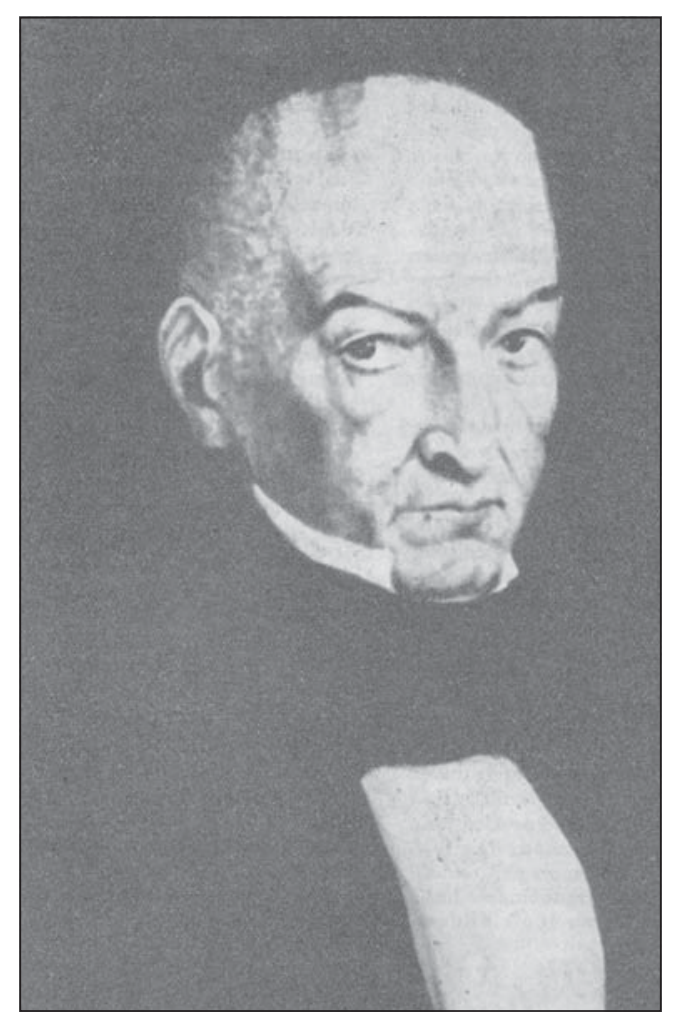

Figura 1. Profesor Pedro Morán.

\section{El doctor Pedro Morán}

Nació en Talca en 1771. Pobre y de una familia sin antecedentes de hidalguía, arrastrando una existencia mísera y oscura durante la Colonia. Aún adolescente se trasladó a Santiago y encontró trabajo como practicante y sangrador en el Hospital San Juan de Dios y al lado del Padre Chaparro se inició en el conocimiento de la anatomía, fisiología y patología clínica, adquiriendo además nociones de cirugía con el doctor Juan Isidro Zapata. Gracias a su extraordinaria inquietud intelectual estudió los textos clásicos de medicina, para lo cual se había preparado aprendiendo latín, francés e inglés.

En 1813 se incorporó al ejército de José Miguel Carrera en calidad de cirujano militar y permaneció durante más de un año en Talca, trabajando en el Hospital Salvador de la ciudad, organizando el servicio sanitario castrense. En marzo de 1814, en Cancha Rayada, cayó prisionero de las tropas de Gabino Gaínza. El tratado de Lircay lo dejó en libertad y se reincorporó al ejército patriota, siguiendo a las tropas a Rancagua. Tomado prisionero y condenado a muerte, debió su salvación a la necesidad de cirujanos que experimentaba el ejercito realista. Fugado de allí se retiró a Santiago, dedicándose a estudiar cirugía.

En 1817 Paroissien, cirujano jefe del Ejército Libertador de los Andes, lo propuso como cirujano militar en grado de teniente y ese mismo año fue designado médico del Hospital San Juan de Dios de Santiago, cargo que mantuvo hasta su muerte.

Solamente en 1821 pudo regularizar su situación para el ejercicio legal de la profesión en el país, mediante examen ante el Protomedicato. Esta circunstancia motivó un serio reclamo de Manuel Grajales, quien hizo caudal de la legitimidad del nacimiento de Morán, el cual tuvo que demostrarla ante la justicia.
El 23 de febrero de 1826, inició cursos privados de anatomía para alumnos que deseaban ser médicos o cirujanos romancistas. Cuando en 1833 se iniciaron los cursos de medicina, tres de ellos se matricularon: Martín Avello, Vicente Mesías y su hijo Bartolomé Morán.

En la ceremonia de inauguración de la primera Escuela en el Instituto Nacional, más arriba mencionada, el doctor Blest al referirse a las cátedras de anatomía y fisiología, manifestó "serán enseñadas con toda exactitud y perfección como en la mejor escuela de Europa por un respetable colega, el doctor Pedro Morán, quien aunque educado en la oscura época de la esclavitud de Chile, aprendió por su ingenio y talento a volar sobre las absurdas doctrinas de la antigüedad y ponerse al nivel de las luces del siglo, fabricándose una reputación que debe adornar las paginas de la historia médica de la patria".

De marzo de 1831 a marzo de 1833, Morán se desempeñó como diputado de la Asamblea Provincial de Santiago.

Debido a que la salud de Morán se encontraba seriamente afectada, no pudo desempeñar la docencia con la continuidad que él hubiera deseado. En 1834 hubo que reemplazarlo transitoriamente su alumno y prosector de anatomía Martín Avello, quien falleció de tuberculosis pulmonar en noviembre del mismo año. Morán reanudó su tarea, pero en 1840 debió sustituirlo su hijo Bartolomé.

El doctor Pedro Morán falleció el 19 de diciembre de 1840, a la edad de 69 años.

Pocas personalidades médicas han sido tan discutidas y vilipendiadas como el doctor Morán. Domingo Amunátegui decía que "era un médico muy desnudo de méritos"; Vicuña Mackenna expresaba que se había educado en Lima, donde había sido lo que en el lenguaje criollo de América se llama "varchilón”, es decir, un curandero que entendía de todo, desde la lanceta hasta el latín y desde la bacía o palangana de las barberías hasta el "ballo" de los muertos y "de cuyo supino y rebuscado pedantismo ha quedado mucha mejor memoria que de sus desaciertos" "-6.

Así mismo el doctor José Grossi escribía: "Pedro Morán, como Bustillos, hijo de sus obras, había estudiado en épocas en que no se daba gran importancia a la anatomía, en que no se enseñaba nada, más propiamente hablando; y en un ramo absolutamente práctico como este, no puede suplir la elocuencia a la preparación y los alumnos de Morán se resentían en los cursos superiores de esta falta de una base tan fundamental en el aprendizaje de las ciencias médicas.

En vista de estos hechos, de que el plan de estudios no guardaba consonancia con el adelanto de las ciencias, hizo que José Vicente Bustillos aconsejara al Gobierno en octubre de 1838, mandar a los alumnos más distinguidos de Medicina a Europa, con el objeto que perfeccionaran sus conocimientos, para consagrarse después a la enseñanza de las ciencias entre nosotros. El Gobierno no atendió las sabias indicaciones del profesor Bustillos y las cosas continuaron como estaban"7.

\section{El doctor Pedro Morán, cirujano militar en la batalla de Rancagua}

La batalla de Rancagua (1 y 2 de octubre de 1814), “tumba gloriosa de la Patria Vieja”, como la llama Julio Bañados Espinosa, término del período histórico, caracterizado por la indecisión de los revolucionarios para tomar francamente el camino de la independencia y por las disensiones civiles que lo perturbaron y que al fin produjeron esa ruda catástrofe ${ }^{8,9}$.

En dicho suceso sangriento le correspondió participar al primer profesor de anatomía de la Escuela Médica de 1833 y cirujano militar, doctor Pedro Morán. Si bien la literatura histórica consultada no nos facultaría para juzgar del todo la actuación del doctor Morán en la batalla de Rancagua, si podríamos al menos calificarla de “dulce y agraz”. 
Eduardo Salas Olano refiere que "Morán es el primer cirujano patriota y su presencia en la acción de Rancagua, en donde fue tomado prisionero en el puesto del deber, es decir, al lado de los mutilados y heridos en defensa de nuestra libertad, son méritos suficientes para poner de relieve su valiente figura; sin embargo, el nombre de Morán es desconocido y los historiadores de la independencia ni siquiera lo mencionan"5.

Pedro Lautaro Ferrer, citando un artículo necrológico del Araucano, del 1 de enero de 1841, dice sobre la batalla de Rancagua que "fue heroico el patriotismo desplegado por Morán, ya exhortando con ardor a sus compañeros, en medio de la pelea a vencer o morir gloriosamente, ya exponiendo a cada paso su vida para asistir a los heridos donde quiera que caían, como pueden acreditarlo varios de sus compañeros sobrevivientes de aquella memorable jornada" ${ }^{10}$.

Laval Manrique anota, a propósito de Pedro Morán, “que una vez más fue hecho prisionero en la batalla de Rancagua en la cual combatió heroicamente. En ella perdió todo su equipaje y su instrumental quirúrgico"4. Agrega Alfonso Asenjo: “Pedro Morán tuvo bajo el mando de O'Higgins una actuación destacadísima en el sitio y desastre de Rancagua. Sus contemporáneos y compañeros de lucha contaban que durante todo el combate alentó a los soldados, asistió a los heridos, armaba ambulancias que luego eran destruidas por los enemigos, infundió ánimo y valor. Cayó herido mientras asaltaba una trinchera junto con el estado mayor de O'Higgins"11.

Veamos ahora la relación sobre la batalla de Rancagua de John Thomas, cuyo verdadero nombre era Thomas Nowlan, que fue secretario del virrey Ambrosio O’Higgins y más tarde de su hijo, alcanzando sobre éste tal ascendiente, después de su destierro, como para sugerirle las ideas y borradores de sus más importantes comunicaciones. La versión exacta al castellano del texto inglés de John Thomas, fue publicada por Carlos Vicuña Mackenna en la Revista Chilena de Historia y Geografía de 1914. Transcribo literalmente el texto en que se menciona al doctor Morán: "el principal cuidado de O'Higgins en todo tiempo era la protección de los heridos. No había, sin embargo, más que un número muy pequeño de cirujanos y uno de ellos, llamado Morán, olvidó hasta tal punto sus deberes, que se escondió tan cuidadosamente como para que transcurrieran varias horas sin encontrarlo."

"O’Higgins, que sentía las heridas de sus valientes como si fueran propias, se indignó con la conducta de Morán, ordenó que se le buscara y llevase a su presencia para juzgarlo y castigarlo. Cuando se le encontró fue enviado a una casa de la plaza que O'Higgins estaba examinando para dedicarla a hospital. Morán y O'Higgins se hallaban uno a cada lado de la puerta, distancia menos de una vara y mientras el primero trataba de excusarse, una bala de cañón pasó entre ambos, con tal aterrador silbido que el pobre médico quedó paralizado de temor. O'Higgins no pudo reprimir una carcajada y le dijo que fuera inmediatamente a cumplir su deber, así podría escapar de las balas de los enemigos, pero que en caso contrario, no escaparía de las balas de sus compañeros, pues lo condenaría inmediatamente a muerte" 12 .

Julio Bañados también aporta lo siguiente: “concluida la tarea, a las dos de la tarde se rompen los fuegos por ambos lados, a la vez que se da comienzo al segundo asalto general de las divisiones realistas. O'Higgins tuvo en esta circunstancia una escapada que el vulgo apellidaría milagrosa y providencial. Estaba cerca del hospital de sangre, censurándole acremente a un cirujano Morán su falta de valor al ocultarse durante el primer ataque, cuando una bala de cañón pasa por entre ambos sin herirlos"”.

Las diferencias entre estos dos informes serían que en el primero no había un hospital de sangre, ya que O’Higgins estaba buscando una casa para transformarla en hospital y en segundo lugar el Director Supremo se ríe a carcajadas de Morán al verlo tan asustado, lo que no menciona Bañados.

Finalmente, en la novela "Bernardo" de Alfredo Sepúlveda, se lee: “en el bando patriota se aprovechó una breve disminución de la refriega para ocuparse de los heridos. No había cirujano. Los heridos fueron llevados a una casa frente a la Iglesia de la Merced. El único médico con que contaba el ejército, Pedro Morán, se había escondido y cuando lo encontraron, mientras Bernardo lo subía y lo bajaba a gritos, una bala de cañón pasó entre ambos: la historia refiere a Bernardo riéndose del incidente y a Morán tiritando de miedo" ${ }^{13}$.

En este último comunicado se dice "que no había cirujano, sino un único médico, Pedro Morán”. Pero reaparece la carcajada de O’Higgins y la casa elegida para hospital. El "pequeño número" de cirujanos de John Thomas, se convierte en "uno" de Alfredo Sepúlveda.

John Thomas, también relata el caso de otro "escondido", que aparentemente no recibió reprimenda: "eran como las siete y media de la mañana del día $1^{\circ}$ de octubre cuando O’Higgins llegó a la plaza de Rancagua. El general Juan José Carrera Verdugo corrió a abrazarlo, declarando que aunque él era el Señor General, se colocaba con toda su división bajo el mando y a las órdenes de O'Higgins, a lo que éste contestó simplemente: Acepto el mando.

Juan José se retiró en seguida a la casa del Cabildo y no salió del agujero en que se escondió hasta la una del día siguiente, cuando oyó que el enemigo se retiraba, como él mismo lo confirmó más tarde"12.

Es necesario destacar que lo relacionado con el doctor Pedro Morán y la batalla de Rancagua, no aparece escrito, ni siquiera el nombre de Morán, en otras obras de historia consultadas de Luis Galdames, Diego Barros Arana, Francisco Antonio Encina y Francisco Frías Valenzuela ${ }^{9,14-16}$. Tan solo en el libro “O’Higgins” de Jaime Eyzaguirre, figura el episodio del encuentro del doctor Pedro Morán con Bernardo O’Higgins, sensiblemente igual al de John Thomas, transcrito más arriba ${ }^{17}$.

Cabría preguntarse: ¿¿el “desastre” de Rancagua fue también el “desastre” de Pedro Morán? Diríamos que no.

Para terminar esta narración, transcribo lo que su alumno Francisco Javier Tocornal pronunció al lado de su tumba: "El anciano respetable, catedrático de anatomía y doctor en Medicina, Pedro Morán, acaba de bajar a la tumba. Un deber sagrado nos ha impuesto la obligación de venir a dejar los restos del chileno benemérito que, no muchos días, apellidábamos nuestro compatriota, nuestro amigo, nuestro maestro. No fue un maestro el que el Supremo Gobierno nos dio en el doctor Morán, sino un padre tan celoso por nuestro adelantamiento que no había para él mayor gloria que el estar rodeado de sus alumnos, franqueándoles sus mismos libros y ofreciendo a instruirlos no solo en las horas de clases, sino en cualquier instante del día.

El sentimiento de separarse para siempre de nosotros, antes de dar cima a nuestra carrera, fue lo que más le amargó en sus postreros momentos.

En el doctor Morán hay que hacer justicia al hombre que de modesta condición social llegó por sus méritos a colocarse en un alto y respetable puesto. Al médico cuyo nombre figura, entre los fundadores de la Escuela Médica Chilena, orlado de timbres honrosos.

Son estos hombres que han vencido en las luchas de la existencia los que deben recordarse perpetuamente, en la vida republicana y en la democracia de la ciencia" ${ }^{\prime \prime}$.

\section{Resumen}

Junto con la fundación de la Escuela Médica de 1833, se destaca la figura del primer profesor de anatomía, fisiología e higiene, doctor Pedro Morán. Además se exponen diversos comentarios sobre su actuación, como cirujano militar en la batalla de Rancagua, unos alabándola y otros mostrando su ocultamiento durante el fragor de la contienda. Se enaltece su origen modesto, competencia y perseverancia en el desempeño de las funciones docentes asignadas. 


\section{Referencias}

1.- Amunátegui, D. El Instituto Nacional. (1835-1845). Impta. Cervantes. Santiago de Chile. 1891.

2.- Costa, C.C. El doctor Guillermo Cunningham Blest. $125^{\circ}$ Aniversario de la fundación de la Escuela Médica de Chile (1833-1958) - Laboratorio Erba Chile S.A.

3.- Laval, M.E. Los estudios y el ejercicio de la medicina en Chile en el primer tercio del siglo XIX. $125^{\circ}$ Aniversario de la fundación de la Escuela Médica de Chile. (1833-1958).Laboratorio Erba Chile S.A.

4.- Laval, M.E. Evolución y desarrollo de la anatomía en Chile. An. Chil Hist Med 1964, 6: 7-75.

5.- Salas, O.E. Historia de la Medicina en Chile. Impta. Vicuña Mackenna. Santiago de Chile. 1894.

6.- Vicuña Mackenna, B. Los médicos de antaño en el Reino de Chile. Impta. de la Librería del Mercurio. Santiago de Chile. 1877.

7.- Grossi J. Reseña del progreso médico en Chile. Impta. de la Opinión. Santiago de Chile. 1895.

8.- Bañados J E. La batalla de Rancagua. Sus antecedentes y sus consecuencias. Ed. Rafael Jover. Santiago de Chile. 1884.

9.- Galdames, L. Estudio de la historia de Chile. Impta. Universitaria. Santiago de Chile. 1911.

10.- Ferrer, P.L. Historia General de la Medicina en Chile. Tomo I. Talca, Chile. Impta. Talca. 1904.

11.- Asenjo, G.A. Pedro Morán. $125^{\circ}$ Aniversario de la fundación de la Escuela Médica de Chile. (1833-1958). Laboratorio Erba Chile S.A.

12.- Thomas, J. La batalla de Rancagua. Rev.Chil.Hist.Geog 1914, 4: 5-59.

13.- Sepúlveda, A. Bernardo. Una biografía de Bernardo O’Higgins. E. B. Chile S.A. Santiago de Chile. 2007.

14.- Barros, A.D. Historia General de Chile. Tomo IX. Ed. Rafael Jover. Santiago de Chile, 1888.

15.- Encina, A.F. Historia de Chile. Desde la Prehistoria hasta 1891. Tomo VI. Ed. Nascimento. Santiago de Chile. 1947.

16.- Frías, V.F. Historia de Chile. Tomo II. Ed. Nascimento. Santiago de Chile. 1976.

17.- Eyzaguirre, J. O’Higgins. Ed. Zig-Zag. 11ª Ed. Santiago de Chile. 1995. 